Classification

Physics Abstracts

$47.90-07.60 \mathrm{~L}-68.15$

\title{
Experimental Investigation of the Hydrodynamic Interactions between a Sphere and a Large Spherical Obstacle
}

\author{
N. Lecoq $\left({ }^{1,2, *}\right)$, F. Feuillebois $\left({ }^{2, *}\right)$, R. Anthore $\left({ }^{1, *}\right)$, C. Petipas $\left({ }^{1, *}\right)$ and F. Bostel $\left({ }^{1, *}\right)$ \\ $\left.{ }^{1}\right)$ UFR des Sciences $\left({ }^{* *}\right), 76821$ Mont Saint Aignan, France \\ $\left({ }^{2}\right)$ Laboratoire d'Aérothermique, CNRS, 4 ter route des Gardes, 92190 Meudon, France
}

(Received 2 June 1994, received in final form 19 October 1994, accepted 24 October 1994)

\begin{abstract}
The hydrodynamic interactions between a spherical particle embedded in a very viscous fluid and a large close spherical obstacle are investigated experimentally. The displacement of the particle is followed by laser interferometry with a $100 \mathrm{~nm}$ resolution. The large obstacle is either convex, plane or concave. The experimental results for the drag coefficient on the particle are compared to the theoretical results written as three terms expansions for small gaps, viz. the result of Cox and Brenner (1967) for a plane obstacle, the result of Jeffrey (1982) and Jeffrey and Onishi (1984) for a convex obstacle and an extension of Cooley and O'Neill (1969) for a concave obstacle. Excellent agreement is found between the experimental and the theoretical results for all cases.
\end{abstract}

\section{Introduction}

The motion of a small particle embedded in a viscous fluid when in the neighbourhood of a large curved obstacle is of interest for various applications: filtration, particle adhesion and reentrainment, etc. If small particles in air or liquids are considered, the Reynolds numbers of the flow relative to the particle and relative to the gap between the particle and the large obstacle may be smaller than unity. This system is modelled experimentally here by using rather large (viz. millimeter size) particles embedded in a very viscous silicon oil.

The technique used here is laser interferometry. The experimental setup and procedure will be described in Section 2. The application of interferometry to the study of hydrodynamic interactions between a particle and walls in a viscous fluid has been presented in a preceding paper by Lecoq et al. [1]. It was then applied to the study of the hydrodynamic interactions between a sphere and a cylinder closed at both ends. The technique allows us to follow the displacement of a particle with a typical resolution of $100 \mathrm{~nm}$. Because of this possibility, it is

$\left({ }^{*}\right)$ The authors are also members of Groupement de Recherche "Physique des Milıeux Hétérogènes Complexes", CNRS.

$\left({ }^{* *}\right)$ URA 808 . 
specially adapted to validate various theoretical results in low Reynolds number hydrodynamics when displacements are small.

The configuration used here consists of a sphere sedimenting in a fluid at rest towards an obstacle at rest. The gap between the sphere and the obstacle is small as compared to the sphere radius. Typical obstacle surfaces are used: (i) a plane; (ii) spherical surfaces with a large radius, viz. a convex one and a concave one.

Theoretical results have been obtained in the literature for the motion of a sphere towards a close plane and a close convex or concave spherical obstacle on the basis of the creeping flow equation of fluid motion, or Stokes equations. Expansions of the friction factor for small gap will be used here. These results will be recalled in Section 3. The expansion valid for a concave obstacle will be extended here.

Our experimental results will then be compared to the theoretical formulae in Section 4 . Finally the conclusion is in Section 5.

\section{Experimental Setup and Procedure}

The experimental setup is sketched in Figure 1.

A spherical particle is sedimenting on the centerline of a closed cylindrical cell filled up with viscous oil. The particle is a steel ball manufactured by SNR. Its mass density is $7800 \mathrm{kgm}^{-3}$. The sphere radius is $3.175 \mathrm{~mm}$. Departure from sphericity is small, typically less than $5 \mu \mathrm{m}$. The surface roughness observed under a scanning microscope is less than $0.5 \mu \mathrm{m}$.

The viscous fluid is silicon oil (Rhodorsyl $47 \mathrm{~V}$ 100000) with kinematic viscosity $\nu=0.1$ $\mathrm{m}^{2} \mathrm{~s}^{-1}$ and mass density $\rho_{\mathrm{p}}=978 \mathrm{kgm}^{-3}$ at $25^{\circ} \mathrm{C}$. Silicon oil was chosen for its very small variation of viscosity with temperature. The very large viscosity was chosen so that fluid inertia effects would be quite negligible as compared to viscous effects. As a matter of fact, calculating the Reynolds number based on the sphere radius and Stokes sedimentation velocity of the particle settling in unbounded fluid at rest, we find that it is of the order of $10^{-5}$.

The measurement cell $\mathrm{C}$ is a closed cylinder with inner height $40 \mathrm{~mm}$ and inner radius 25 $\mathrm{mm}$. The axis of the cylinder is vertical. One cylinder end is closed by a plane glass window $\mathrm{G}$

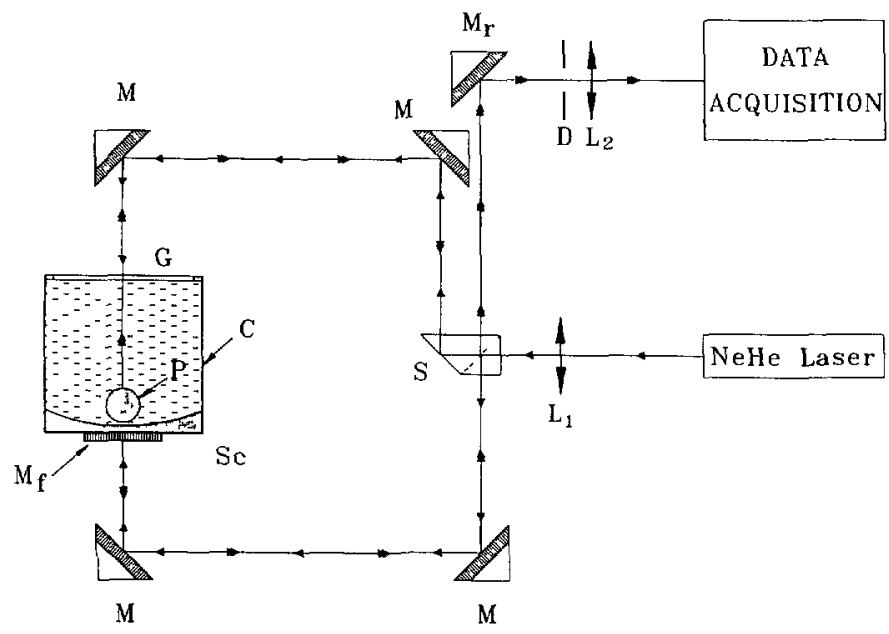

Fig. 1 - Experimental setup. $\mathrm{L}_{1}, \mathrm{~L}_{2}$. Lens; $\mathrm{M}_{\mathrm{f}}$ : Fixed mirror; $\mathrm{M}, \mathrm{M}_{\mathrm{r}}$ : Mirrors; $\mathrm{S} \cdot$ Beam splitter; D: Diaphragm; G: Plane glass window; $S_{c}$ : Curved surface, C: Measurement cell; P: Particle 
of optical quality with thickness $3.00 \pm 0.02 \mathrm{~mm}$. The other end is closed by a curved (either convex or concave) surface, that is a lens of optical quality (made by Newport), with radius $51.68 \mathrm{~mm}$. Both outer end planes of the cell are made parallel to within a tolerance of 5 minutes of arc. This cell arrangement allows us to study the motion of a sphere towards a plane and a curved surface successively by turning the cell upside down. Alternatively, the curve surface may be used before the plane one; this choice does not affect the final result since the characteristics of the fluid do not vary during the experiment. First, there is no temperature variation during the experiment: the absorption of laser energy is very small and the heat dissipated by viscous stresses is small as well. Secondly, the oil is composed of dimethyl siloxane chains of various lengths so that even if some large chains are broken by the particle motion, the overall distribution of chains is not much affected.

Before starting the experiment, the steel ball is maintained at the upper side of the container with a magnet located outside the cell, on top of it. At the beginning of the experiment, the magnet is taken away and the ball starts settling. The departure of the ball was found to be quite smooth, because of the low sedimentation velocity, especially near the wall.

A $15 \mathrm{~mW}$ He-Ne laser beam with wavelength $\lambda=632.8 \mathrm{~nm}$ is focused at the center of the cell with a lens $\mathrm{L}_{1}$. It is then divided by a beam splitter into two separate beams having roughly the same power. Both beams are then deviated by successive reflections onto mirrors $\mathrm{M}$, following symmetrical paths. In the preceding version of the experiment [1], the two beams were reflected by the particle; it was then necessary for the beams to follow symmetrical paths with nearly equal lengths. In the present version of the experiment, only one of the beams is reflected by the particle in motion. The other beam is a fixed reference one. Since this reference beam may in principle have any length, we chose a simple setup in which it is reflected by a fixed plane mirror $M_{f}$ attached to the other end of the cell. This choice allows us to switch from one setup to the other when necessary, by simply changing the cell.

Both reflected beams then follow the same paths in the reverse direction, up to the separator where they combine to form interference fringes. The resulting beam is extracted from the interferometer by the mirror $M_{r}$. It is then stopped down and focused onto the end of an optical fiber coupled to a photomultiplier. The use of an optical fiber makes it easier to select the useful central part of the reflected beam.

The displacement of the particle results in a shifting of the interference fringes which appear as concentric clear and dark rings. Shifting from a dark to the next clear fringe (or conversely) is characteristic of a displacement $\Delta z$ of the particle such that

$$
\Delta z=\frac{\lambda}{4 n}
$$

in which $n$ is the index of refraction of the liquid for the given wavelength $\lambda=632.8 \mathrm{~nm}$, that is $n=1.404$ for the silicon oil. Thus the absolute resolution of the apparatus, that is the smallest displacement which can be measured with a good confidence, is $\Delta z \simeq 100 \mathrm{~nm}$.

Note that in the setup used in [1] the cylinder was closed by plane optical windows at both ends. Then both beams were reflected by the particle so that the sensitivity was $\lambda /(8 n) \simeq$ $50 \mathrm{~nm}$ that is two times smaller. The twofold increase in the sensitivity when changing to the present setup is in a sense the price to pay for using any type of obstacle.

The signal intensity detected by the photomultiplier varies as a function of the particle displacement $z$ according to the following formula

$$
I=I_{0}\left[\cos \left(\frac{2 \pi n z+\phi}{\lambda}\right)\right]^{2}
$$


where $\phi$ is an unknown constant phase shift which depends on the various reflections applied to both beams.

This interferometric signal is converted into an electric signal by the photomultiplier; this apparatus manufactured by Hamamatsu has a bandwidth in wavelength $300 \mathrm{~nm}<\lambda<850$ nm. The analog signal is transformed into a digital one with a converter board installed in a $\mathrm{PC}$ compatible microcomputer. Data acquisition programs are written in $\mathrm{C}$ language with some parts in assembler language to increase data acquisition frequency.

The results in [1] were relative to the motion of a spherical particle along its whole trajectory inside the cell. The present article is only concerned with the approach of a particle to a wall. Thus the data acquisition is started when the sphere comes close to the wall, at a distance of the order of $200 \mu \mathrm{m}$ from it. The beginning of the acquisition of the electric signal is synchronized with the value $300 \mathrm{~Hz}$ of the shift frequency of the interference fringes. A typical signal is presented in Figure 2. It corresponds to a sphere approaching a concave obstacle.

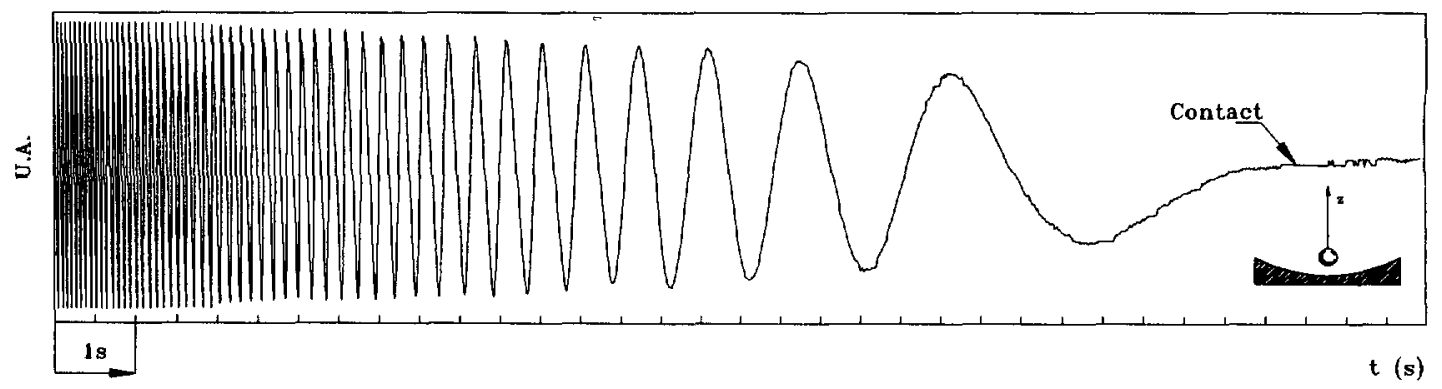

Fig. 2. - Example of an interferometric signal for a spherical particle approaching a concave obstacle. The beginning of the signal corresponds to a particle velocity of the order of $3 \mu \mathrm{m} / \mathrm{s}$.

The recorded signal is then processed in the microcomputer as follows. The average line is calculated first. The successive times at which the signal crosses this average line are then obtained. The difference between two such times is the elapsed time interval $\Delta t$ for the particle to move a distance $\Delta z$, Equation (1). The particle velocity is then simply calculated as

$$
w_{\mathbf{p}}=\Delta z / \Delta t
$$

Because of the small size of $\Delta z$ and of the excellent precision on the measurement of time, it was found that this simple method is sufficient to provide a smoothly varying velocity. As indicated above, the data aquisition is started for the value $300 \mathrm{~Hz}$ of the shift frequency of the interference fringes; this corresponds to a sphere velocity of $67.10 \mu \mathrm{m} / \mathrm{s}$.

The contact position for which the particle velocity vanishes is clearly visible on the signal curves. It is obtained with a $100 \mathrm{~nm}$ precision. This contact position is taken as the origin of the sphere-to-wall distances. The experimental gap between the particle and the obstacle based on this origin is then reconstructed at the end of each experiment.

Thus, a practically continuous set of particle positions and velocities can be recorded.

The present setup is adapted to study the purely one-dimensional motion of a sphere along the vertical. The verticality of the upper beam is adjusted by forming an autocollimation of the beam on a surface of mercury. The lower beam is adjusted by superimposing it to the upper one. The cell is then made horizontal by autocollimation of the upper beam on an 
optical mirror attached to the upper side of the cell. That is, the setup is designed in such a way that any horizontal motion would be quite small, and if present, would be immediately detected: interferences with three waves would then form and the signal would be unusable. In the case of a concave or a convex wall, the axis of symmetry has to be exactly vertical and cross the sphere centre during its motion. The perfect verticality of the axis of symmetry is made possible by precise construction of the cell, as detailed above. For a concave wall, the sphere if off axis is then moving towards the axis of symmetry. This observation agrees with the theoretical prediction of Falade and Brenner [2]. Conversely for a convex wall, in the case of a bad alignment, the closer the sphere from the wall, the larger the discrepancy. The sphere is then rapidly moving out of the beam and the interferometric signal is lost. It is thus easy to ascertain the position of the sphere so as to measure its vertical velocity.

\section{Theoretical Formulae for Two Close Converging Spherical Surfaces}

3.1. Problem and Notation. - Typical obstacles chosen in the experiment are a plane and a spherical surface with a large radius.

Consider then (Fig. 3) a sphere with radius $a$ moving with velocity $w_{\mathrm{p}}$ towards a large spherical obstacle at rest. Let $\left|a_{2}\right|$ be the radius of the particle and let $\kappa=-a / a_{2}$. By definition, $a_{2}>0, \kappa<0$ for a convex obstacle, and $a_{2}<0, \kappa>0$ for a concave obstacle. Let $z$ be the axis along the line of centers of the spheres. Let $\epsilon a$ be the gap between the surfaces of the spheres. We assume $\epsilon \ll 1$.

Solving Stokes creeping flow equations of fluid motion, the expression for the drag force on the sphere is obtained as linear function of the velocity $w_{\mathrm{p}}$ in the form

$$
F_{z}=-6 \pi a \mu w_{\mathrm{p}} f_{z z}^{\mathrm{T}}
$$

The force is along $z$ by symmetry, as indicated by the subscript $z . f_{z z}^{\mathrm{T}}$ is the friction coefficient relating the force in the $z$ direction to the translational velocity in the $z$ direction. $\mu$ is the dynamic viscosity of the fluid.

3.2. Plane Obstacle. - An exact theoretical result for $f_{z z}^{\mathrm{T}}$ for the motion of a sphere towards a plane was derived independently by Brenner [3] and Maude [4] using the method of bipolar coordinates. A three term expansion of this result for small gaps $(\epsilon \ll 1)$ was later obtained by Cox and Brenner [5] and independently by Cooley and O'Neill [6]:

$$
f_{z z}^{\mathrm{T}}=\frac{1}{\epsilon}-\frac{1}{5} \log \epsilon+0.9713
$$

Note that other terms involving the fluid inertia were also derived by Cox and Brenner [5], but these terms will be neglected here. There is a small discrepancy between the two quoted papers in that Cox and Brenner obtained the more precise value 0.971264 for the constant term, whereas Cooley and O'Neill obtained the value 0.971280. Since the next term in the expansion is of order $\epsilon \log \epsilon$, the difference between both values would be significant only if $|\epsilon \log \epsilon|<10^{-5}$ which occurs for $\epsilon<10^{-6}$. For a particle with a radius of the size of a few millimeters, the gap would then be of the order of a few nanometers. Such small gaps are not considered in the present experiment since the sensitivity is $100 \mathrm{~nm}$. The value 0.9713 which we use in Equation (4) is compatible with the results of Cox and Brenner [5] and Cooley and O'Neill [6] and it is sufficient for experimental purposes.

It is also of interest to compare expansion (4) to the exact result of Brenner [3] and Maude [4]. As shown in [6] the two results differ by less than $0.3 \%$ when $\epsilon=0.12763$ which is above the non-dimensional gaps used in the experiment. Thus the expansion (4) is sufficient for our purposes 

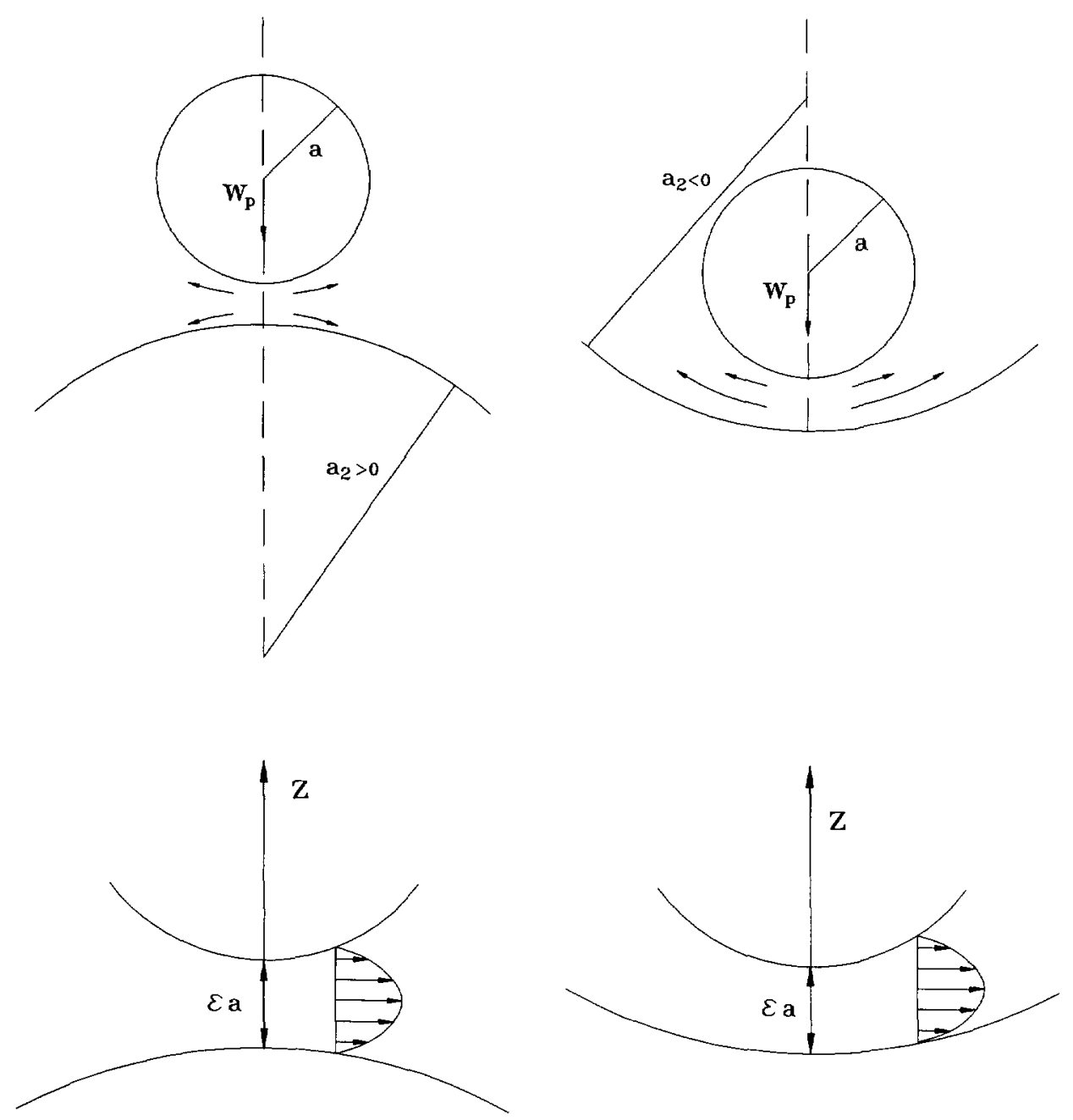

Fig. 3 - Notation for a spherical particle moving towards a large spherical obstacle. In the experiment, $a=3175 \mathrm{~mm},\left|a_{2}\right|=|a / \kappa|=51.68 \mathrm{~mm}$.

3.3. Spherical Convex Obstacle. - Cooley and O'Neill [6] also obtained an expression for the friction coefficient on a sphere moving towards a close spherical obstacle at rest. Their result is in the form of a two-term expansion for small gaps:

$$
f_{z z}^{\mathrm{T}}=\frac{1}{(1-\kappa)^{2} \epsilon}-\frac{\left(1-7 \kappa+\kappa^{2}\right)}{5(1-\kappa)^{3}} \log \epsilon+O(1)
$$

Note that there is a misprint in Equation (7.12) of [6].

This result was later generalized for a convex obstacle of radius $-a / \kappa$, with $\kappa<0$, by Jeffrey [7] who derived an expansion up to order $O(\epsilon)$ (his Eq. (5.9)). For our purpose of 
Table I. - Values of $K(\kappa)$.

\begin{tabular}{|c|c|c|c|}
\hline$\kappa$ & 0 & 0.1 & 0.2 \\
\hline$K(\kappa)$ & 0.9713 & 1.1378 & 1.504 \\
\hline
\end{tabular}

comparing with the present experiments, we retain only terms up to order $O(1)$ :

$$
f_{z z}^{\mathrm{T}}=\frac{1}{(1-\kappa)^{2} \epsilon}-\frac{\left(1-7 \kappa+\kappa^{2}\right)}{5(1-\kappa)^{3}} \log \epsilon+K(\kappa)
$$

The function $K(\kappa)$, obtained by matching expansion (6) with the exact solution for two spheres, was tabulated by Jeffrey and Onishi [8]: see their Table II in which $\lambda$ is $-\kappa$ and $A_{11}^{\mathrm{X}}$ is $K$. Interpolating between these values, we obtain $K=1.0192$ for the value $\kappa=-0.0615$ used in the experiment.

3.4. Spherical Concave Obstacle. - Expansion (5) obtained by Cooley and O'Neill [6] is also valid for a sphere and a close concave obstacle of radius $a / \kappa$ with $\kappa>0$. Following then the approach of Jeffrey [7] with now $\kappa>0$, expansion (6) still holds. The function $K(\kappa)$ will be obtained by fitting expansion (6) to the exact solution for two spheres.

From the linearity of Stokes equations of fluid motion, the exact expression for the force on the sphere moving with velocity $w_{\mathrm{p}}$ towards the obstacle at rest is obtained as the sum of the forces for the following two cases:

(i) the force on the sphere moving with velocity $w_{\mathrm{p}} / 2$ towards the obstacle moving with an equal and opposite velocity $-w_{\mathbf{p}} / 2$;

(ii) the force on the sphere moving with velocity $w_{\mathrm{p}} / 2$ when the obstacle moves with the same velocity $w_{\mathrm{p}} / 2$. As remarked by Cooley and O'Neill [6], this is a solid body motion (the fluid is at rest in the frame moving with velocity $w_{\mathrm{p}} / 2$ ) so that the force on either wall is zero.

The required force is then

$$
F_{z}=-6 \pi a \mu\left(w_{\mathrm{p}} / 2\right) F_{1}
$$

in which $F_{1}$ is the friction coefficient for case (i). This coefficient is tabulated by Cooley and O'Neill [6] in their Table III. Thus from (3) the exact value of the friction coefficient for a sphere moving towards a close concave obstacle is obtained as $\left(f_{z z}^{\mathrm{T}}\right)_{\mathrm{ex}}=F_{1} / 2$.

The function $K(\kappa)$ is then obtained by fitting expansion (6) to the exact solution $\left(f_{z z}^{\mathrm{T}}\right)_{\mathbf{e x}}$, that is, denoting $\left(f_{z z}^{\mathrm{T}}\right)_{\text {ap }}$ the sum of the first two terms in (6),

$$
K(\kappa)=\left(f_{z z}^{\mathrm{T}}\right)_{\mathrm{ex}}-\left(f_{z z}^{\mathrm{T}}\right)_{\mathrm{ap}} .
$$

The result is given in Table I. By interpolating these values, we obtain $K=1.0499$ for the value $\kappa=0.0615$ used in the experiment.

3.5. Relative Importance of the Terms in the Expansions. - Rewriting expansion (6) for small gaps as

$$
f_{z z}^{\mathrm{T}}=\frac{M(\kappa)}{\epsilon}+L(\kappa) \log \epsilon+K(\kappa)
$$

the relative significance of these three terms is presented in Figure 4 by plotting 




Fig. 4 - Plot of $1 / f_{z z}^{\mathrm{T}}$ showing the relative significance of the terms in expansion (9) of $f_{z z}^{\mathrm{T}}$, for the three cases of a convex surface with $\kappa=-0.0615$ (top three lines), a plane (middle three lines), and a concave surface with $\kappa=0.0615$ (bottom three lines). The solid lines represent the first term in the expansions, the dash-dotted lines represent the first two terms and the dashed lines the three terms.

- $\left[\frac{M(\kappa)}{\epsilon}\right]^{-1}$ (solid line)

- $\left[\frac{M(\kappa)}{\epsilon}+L(\kappa) \log \epsilon\right]^{-1}$ (dash-dotted line),

- $\left[\frac{M(\kappa)}{\epsilon}+L(\kappa) \log \epsilon+K(\kappa)\right]^{-1}$ (dashed line)

versus the non-dimensional gap $\epsilon$. These plots are provided for the three cases of a plane, a convex surface with $\kappa=-0.0615$ and a concave surface with $\kappa=0.06$.

\section{Results and Discussion}

Using the experimental velocity $w_{\mathrm{p}}$ of the particle, and introducing its weight and buoyancy together with expression (3) for the drag force into Newton's law of motion, the experimental friction coefficient $f_{z z}^{\mathrm{T}}$ is obtained. The particle inertia, which is of the same order as the fluid inertia, is negligible for the small Reynolds numbers considered here.

The experimental friction coefficient $f_{z z}^{T}$ for the motion of a sphere towards a plane wall is represented versus the non-dimensional gap $\epsilon$ in Figure 5 as a logarithmic plot for $\epsilon a \leq$ $200 \mu \mathrm{m}$. The agreement with the theoretical expansion (4) is excellent. The fluctuations due 


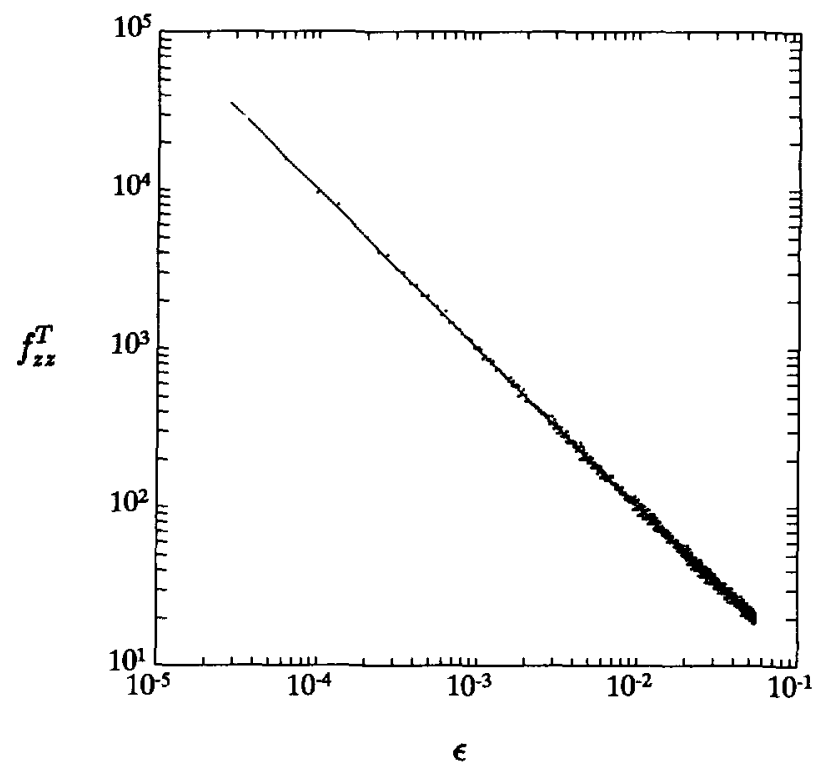

Fig 5. - Logarithmic plot of the friction factor versus the non-dimensional gap for a sphere moving towards a plane wall and in a close vicinity to it; experiment and theory.

to temperature variations are negligible. The small discrepancy observed for the smallest gaps is the consequence of the presence of a small roughness on the sphere. It can be checked that for the small $\epsilon$ for which the discrepancy occurs, $\epsilon a$ is of the order of the roughness. It is estimated here to be $0.5 \mu \mathrm{m}$, equal to the maximum roughness $0.5 \mu \mathrm{m}$ observed under the scanning microscope (Sect. 2).

Defining the Stokes sedimentation velocity of the particle in unbounded fluid at rest as

$$
w_{\mathrm{ps}}=-\frac{F_{z}}{6 \pi a \mu}
$$

the non-dimensional particle velocity is obtained as

$$
\frac{w_{\mathrm{p}}}{w_{\mathrm{ps}}}=\frac{1}{f_{z z}^{\mathrm{T}}}
$$

The results can be alternatively plotted in terms of the non-dimensional velocity $w_{\mathrm{p}} / w_{\mathrm{ps}}$ versus the non-dimensional gap $\epsilon$. They are shown in Figure 6.

The experimental non-dimensional velocity of the sphere moving towards a curved obstacle is also shown in Figure 6. It is compared to the theoretical value obtained from (11) and the expression for the friction coefficient from Section 3.3 and Section 3.4 for a convex and concave obstacle, respectively.

The agreement between experiment and theory is excellent for the plane as well as for the curved obstacles.

It is seen in the Figure that the curvature of the obstacle strongly modifies the sphere velocity. This is because the flow of liquid out of the region near the contact point is responsible for the drag force; and this liquid flow is strongly affected by the curvature of the obstacle. When the surface is convex, the outside flow is made easier and the drag decreases; the reverse happens 


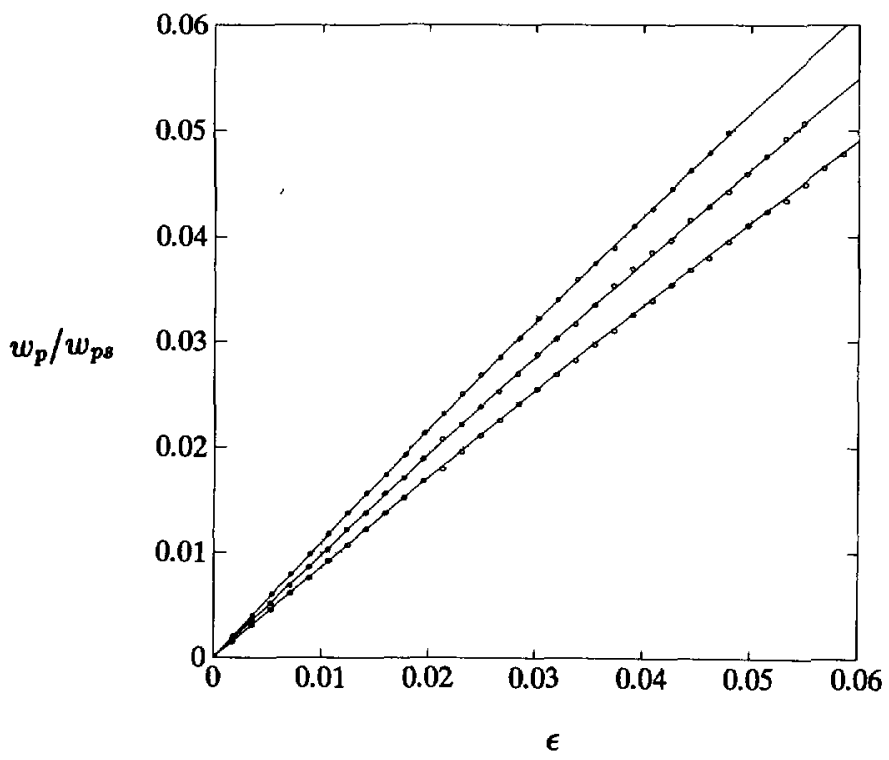

Fig. 6. - Plot of the non-dimensional velocity versus the non-dimensional gap for a sphere moving towards a convex spherical surface (top), a plane (middle), and a concave spherical surface (bottom). The sphere is in close vicinity to the obstacle. Experiment (circles) and theory (solid lines).

when the surface is concave. It is also seen in the Figure that the case of a plane obstacle is intermediate between the convex and concave surfaces, as expected.

The influence of the surface curvature increases for small gaps: the relative difference between the experimental curves for the convex and concave obstacles is of the order of $20 \%$ for $\epsilon \simeq$ $5 \times 10^{-2}$ and $25 \%$ for $\epsilon \simeq 3 \times 10^{-3}$. Inspecting the curves in Figure 6, one sees that the experimental results for the convex or the concave surface separate continuously from those for the plane surface.

It is interesting to note that the velocity ratios $\left(w_{\mathrm{p}}\right)_{\text {convex }} /\left(w_{\mathrm{p}}\right)_{\mathrm{plane}}$ and $\left(w_{\mathrm{p}}\right)_{\text {concave }} /\left(w_{\mathrm{p}}\right)_{\text {plane }}$ are practically constant over the whole experimental range. This can be understood by recasting expansion (6) for a curved obstacle in the following form:

$$
f_{z z}^{\mathrm{T}}=\frac{1}{(1-\kappa)^{2}}\left[\frac{1}{\epsilon}-\frac{1}{5} \log \epsilon+0.9713+\frac{6 \kappa}{5} \log \epsilon+O(K(\kappa)-0.9713)\right] .
$$

The first three terms in brackets are the expansion terms for the plane equation (4). The fourth term is small as compared to $1 / \epsilon$ for small $\kappa$ : for $\kappa=0.0615$ and $\epsilon=0.06$, we obtain

$$
\epsilon\left(\frac{6 \kappa}{5} \log \epsilon\right)=1.25 \%
$$

The fifth term in brackets in (12) is $O\left(5 \times 10^{-2}\right)$, that is very small as compared to $1 / \epsilon$. Thus it is seen that the three-term expansion of the friction factor for a curved (convex or concave) obstacle with a large radius can be obtained by multiplying the corresponding expansion for the plane by $1 /(1-\kappa)^{2}$.

The region of small gaps $\epsilon \leq 0.01$ is replotted in more detail in Figure 7. The experimental 


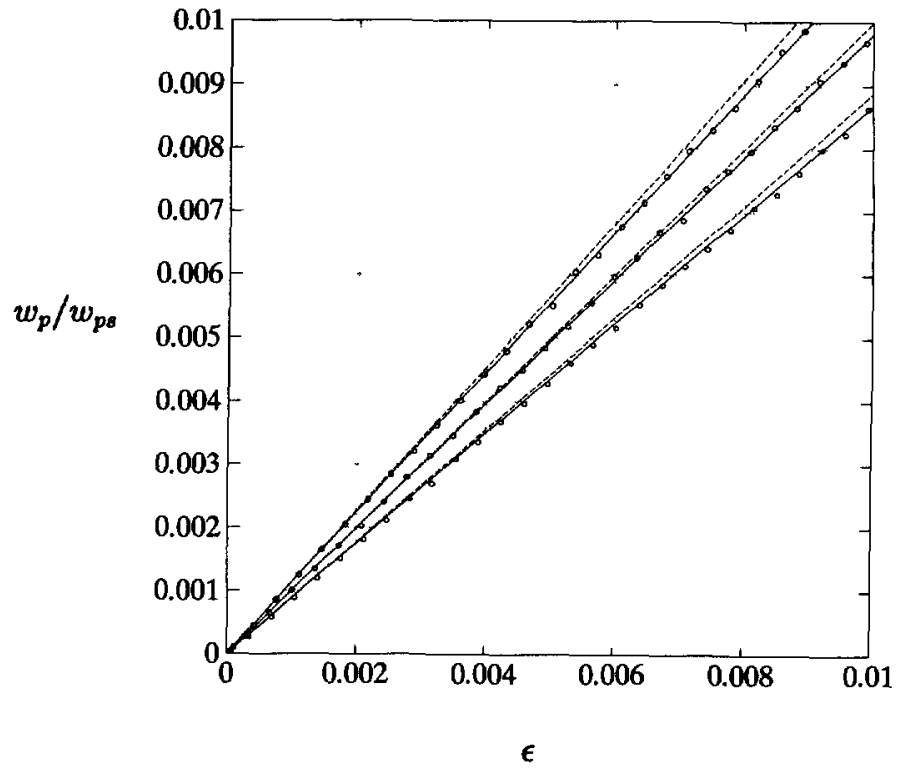

Fig. 7. - Same as in Figure 6. Details of the region of small gaps $\epsilon \leq 0.01$. The lubrication law is also shown as dashed straight lines for the three cases of a convex $(\kappa<0)$, plane $(\kappa=0)$ and concave $(\kappa>0)$ obstacle.

data can be compared to the lubrication law, which is written

$$
\frac{w_{\mathrm{p}}}{w_{\mathrm{ps}}}=(1-\kappa)^{2} \epsilon
$$

using (11) and the first term in (5). In Figure 7 one can see that (13) only applies for small non-dimensional gaps, roughly $\epsilon \leq 0.005$, that is for a sphere-to-wall distance less than or equal to $15 \mu \mathrm{m}$.

The relative hydrodynamic effects of the other walls of the cell are negligible. This can be checked using the characteristic dimension $R=25 \mathrm{~mm}$ for the cylinder radius: the relative effect of the lateral walls is $O((a / R) /(1 / \epsilon))$; for the largest value $\epsilon=0.06$ used in the experiment, this is $0.76 \%$.

\section{Conclusion}

The hydrodynamic interactions of a spherical particle with a close obstacle in creeping flow have been measured with laser interferometry. The typical obstacles considered here are a plane obstacle, a convex obstacle and a concave spherical obstacle with large radii. The experimental results for the friction coefficient are in excellent agreement with the theoretical results written as a three term expansion for small gaps.

The interferometric technique is well-adapted to validate theoretical results for the drag on a particle obtained from the creeping flow equations, especially for small displacements when other methods would not be so efficient. 


\section{References}

[1] Lecoq N., Feuillebois F., Anthore N., Anthore R., Bostel F. and Petipas C., Precise measurement of particle-wall hydrodynamic interactions at low Reynolds number using laser interferometry, Phys. Fluıds A 5 (1993) 3-12.

[2] Falade A. and Brenner H., First-order wall curvature effects upon the Stokes resistance of a spherical particle moving in close proximity to a solid wall, J. Flurd Mech. 193 (1988) 533-568.

[3] Brenner H., The slow motion of a sphere through a viscous fluid towards a plane surface, Chem. Eng. Scr. 16 (1961) 242-251.

[4] Maude A.D., End effects in a falling-sphere viscometer, Bratısh J. Appl. Physics 12 (1961) 293295.

[5] Cox R.G. and Brenner H., The slow motion of a sphere through a viscous fluid towards a plane surface II. Small gap widths including inertial effects, Chem. Eng. Sci. 22 (1967) 1753-1777.

[6] Cooley M.D.A. and O'Neill M.E., On the slow motion generated in a viscous fluid by the approach of a sphere to a plane wall or stationary sphere, Mathematika 16 (1969) 37-49.

[7] Jeffrey D.J., Low-Reynolds-number flow between converging spheres, Mathematika 16 (1982) 106-121.

[8] Jeffrey D.J. and Onishi Y., Calculation of the resistance and mobility functions for two unequal rigid spheres in low-Reynolds-number flow, J. Flund Mech. (UK) 139 (1984) 261-290. 